\title{
The evolution of the welded joints structure at low cycle fatigue
}

\author{
Ekaterina Poyarkova ${ }^{1, *}$, and Iskander Kuzeev ${ }^{2}$ \\ ${ }^{1}$ Orenburg State University, Orenburg, Russia \\ ${ }^{2}$ Ufa State Petroleum Technological University, Ufa, Russia
}

\begin{abstract}
The effect of cyclic damage on the structure evolution and the change in the micro stresses of the crystal cell in characteristic zones of dissimilar welded joints of steels strength classes X60 and X70 is estimated. It is shown, that with increase in the number of loading cycles in less strong metal of the welded joint, relaxation of internal stresses, realized by splitting individual ambits of ferrite grain or by the initiation of micro cracks, is observed. The results of fractographic analysis of a less reliable weld zone at different levels of samples fractures of accumulated damage are presented.
\end{abstract}

\section{Introduction}

Trends in the development of the modern oil and gas industry are due to the impressive volume of requirements growth for the survivability of structures as full-fledged constructions. Their individual components from the diverse positions of the need to ensure longterm and trouble-free operation. The presence of welded joints in such structures is inevitable.

Since the process of development and accumulation of damages both of structures materials in general, and of their welded joints in particular, is localized; the resource of the entire construction, according to [1], is determined by the "resource of their critical zones". Such localization is caused by the presence of stress concentrators and a combination of complex interactions of various competing factors, among which it is possible to isolate selectively the evolution of the structure at different scale levels, the relaxation of stresses and the intensity of manifestations in the weakened places of the construction associated with their geometric features.

The issues of increasing the safety of operation of critical engineering facilities by preventing fragile destruction of their elements are becoming more urgent and acute every year. This is true for oil and gas pipelines that have heterogeneous butt joints. One of the reasons for their destruction is internal structural and mechanical changes occurring in the metal.

The purpose of this work was to assess the effect of cyclic damage on the trajectory of the evolution of structural heterogeneity of dissimilar welded joints of low-carbon steels for welded structures of 09G2FB and $17 \mathrm{G} 1 \mathrm{SU}$.

\section{Materials and methods of research}

To analyze the structural changes in dissimilar welded joints in the accumulation of cyclic damages, low-carbon steels for welded structures were chosen as the most commonly used in the pipeline systems of the oil and gas industry of the Russian Federation, grades 09G2FB (strength class X70) and 17G1SU (strength class X60). The chemical composition of these steels is regulated by normative documents for the material and is given in Tables 1 and 2 .

Table 1. The chemical composition of structural steel 09G2FB for TS 13657842-150-2006, mas. \%

\begin{tabular}{|l|l|l|l|}
\hline $\mathbf{C}$ & $<0,12$ & $\mathbf{C u}$ & $<0,3$ \\
\hline $\mathbf{S i}$ & $0,17-0,37$ & $\mathbf{P}$ & $<0,035$ \\
\hline $\mathbf{M n}$ & $1,4-1,8$ & $\mathbf{S}$ & $<0,035$ \\
\hline $\mathbf{C r}$ & $<0,3$ & $\mathbf{A}$ & $<0,008$ \\
\hline $\mathbf{N b}$ & $0,7-0,9$ & $\mathbf{V}$ & $0,8-0,9$ \\
\hline
\end{tabular}

Table 2. Chemical composition of structural steel 17G1SU according to GOST 5520-79, mas. \%

\begin{tabular}{|l|l|l|l|}
\hline $\mathbf{C}$ & $<0,17$ & $\mathbf{C u}$ & $<0,3$ \\
\hline $\mathbf{S i}$ & $0,4-0,6$ & $\mathbf{P}$ & $<0,035$ \\
\hline $\mathbf{M n}$ & $1,15-1,6$ & $\mathbf{S}$ & $<0,04$ \\
\hline $\mathbf{C r}$ & $<0,3$ & $\mathbf{A}$ & $<0,008$ \\
\hline Nb & $<0,3$ & $\mathbf{V}$ & $<0,08$ \\
\hline
\end{tabular}

The reliability of obtained results was ensured by the use of welded samples with a thickness correlates with real sizes of devices, produced according to GOST 25502-79. Welded samples were obtained by manual electric arc welding (direct current with a current strength of 80-100 A, an electrode of the UONI-13/55 brand with a diameter of $4 \mathrm{~mm}$ ) of two plates of sheet products from the above-mentioned steels of increased strength. The direction of the samples cutting (along the sheet) was selected from the loading conditions of the products and the technology for material obtaining.

Durability of dissimilar welded joints from steels in the low-cycle region was determined under cyclic elastoplastic loading [2, 3]. As a test machine, the facility of IR 5113-100 was used. The number of loading cycles was set at $0.2 ; 0.4 ; 0.6$ and 0.8 from the

* Corresponding author: yarko.e@mail.ru 
destructive $\left(\mathrm{N}_{\mathrm{i}} / \mathrm{N}_{\mathrm{p}}\right)$. The characteristic zones of the investigated welded joints were the zones of the main metals forming part of the welded joint, the central zone of the weld metal and the zone of thermal influence on the side of both metals.

Metallographic analysis of dissimilar welded joints 09G2FB-17G1SU at different levels of metal accumulation of cyclic damage was performed on optical microscopes Neophot-2, AxioObserver.Dlm and using a scanning electron microscope JEOL JSM-6460LV with a console of energy-dispersive analysis INCA-energy in reflected and secondary electrons at accelerating voltage 25 кВ. Grain evaluation was carried out by comparison with scales in accordance with GOST 5639-82.

Glagolev (point) and Rosival (linear) methods performed the quantitative evaluation of the steels phase composition in welded joints. The ratio of ferrite and perlite in various zones of welded joints was determined with the help of the image analysis program by the automatic evaluation method "SIAMS 700".

Defects in the crystal structure in various zones of welded joints were studied by X-ray diffraction on DRON-3 and DRON-UM1 diffractometers in iron radiation at a voltage of $30 \mathrm{kV}$ and a current value of 20 $\mathrm{mA}$ in the range of angles $2 \theta$ from 18 to $90^{\circ}$ in steps $\Delta(2 \theta)=0.02^{\circ}$ and an exposure of $10 \mathrm{~s}$ at a point. The micro deformations of the cells are determined. The dislocation density values in the characteristic zones of the investigated samples of dissimilar welded joints are calculated. The results were processed using a program for the analysis and visualization of scientific and statistical data SigmaPlot.

\section{The results of the study and their discussion}

\subsection{Metallographic analysis of a heterogeneous welded joint of steel 09G2FB-17G1SU}

As is known, the steels $09 \mathrm{G} 2 \mathrm{FB}$ and $17 \mathrm{G} 1 \mathrm{SU}$ of the investigated welded joint refer to low-carbon, lowalloyed, with ferritic-perlite structure welded without restrictions. In the heat-affected zone of these steels, martensitic decomposition is almost completely absent. Welding of such steels does not cause any difficulties.

The initial structural state of the investigated welded joints was studied by scanning electron microscopy; the results are shown in Fig. 1. The structures of the welded joint throughout the section are ferrite-pearlite $(\mathrm{F}+\mathrm{P})$ mixtures whose morphologies depend on the area of the welded joint. In the microstructure of the base metal 9G2FB a segregation banding that coincides with the direction of sheet is observed. Large grains of sorbitollike perlite $(\mathrm{P})$ and sections of the ferritic $(\mathrm{F})$ grid, which have an equiaxed and obvious dendritic structure, represent the weld metal. The cross-section of the grains undergoes a significant change with distance from the smelting boundary, which is due to the growth of the crystals from the smelting line towards the heat removal in the direction of displacement of the heat source. The microstructure of the base metal of grade $17 \mathrm{G} 1 \mathrm{SU}$ also has a structure - F $+\mathrm{P}$, obvious segregation banding is observed. The grains have a flattened elongated shape due to technological features of the sheet method of production.

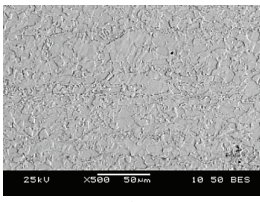

A)

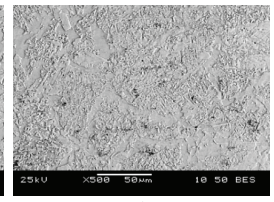

B)

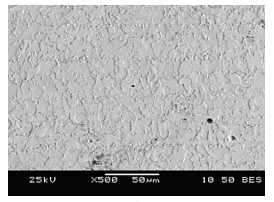

C)
Fig.1. Microstructures of characteristic zones of heterogeneous welded joint: A) the base metal 09G2FB; B) the metal of the weld center; C) the base metal of $17 \mathrm{G} 1 \mathrm{SU}$

In the area of the transition zone from the base metal 09G2FB to the seam, the microstructure is identified as a ferrite-carbide mixture (Fig. 2, a). Microstructure of the transition zone from the seam to the base metal 17G1SU- F+C (ferrite-cementite) mixture (Fig. 2, b).

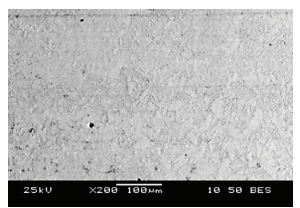

A)

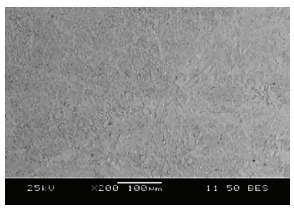

B)
Fig. 2. Microstructures of zones of thermal influence of a heterogeneous welded joint: A) HAZ from the steel side 09G2FB; B) HAZ from the steel side 17G1SU

The ratio of ferrite and perlite in the characteristic zones of the welded joints, established with the help of the SIAMS 700-image analysis program, made it possible to note a marked reduction in the percentage of the pearlitic component at the center of the seam relative to the base metal of the stronger material (steel grade X70 of 09G2FB). The growth of thermal influence of both steels was observed in these zones. It was found that the micro hardness of ferrite varies insignificantly across all sections of the welded joint. The micro hardness of perlite grows in the areas of the welded joint with increased percentage of it.

\subsection{Analysis of structural changes of dissimilar welded joints under cyclic damage}

Fig. 3 shows the evolutionary changes in the steel microstructure over the welded joint cross section with the accumulation of cyclic damage at the mesoscale structural level.

In the process of cyclic loading, the structures of all characteristic zones of a heterogeneous weld joint undergo some changes. At the initial stage of damage accumulation (level $\mathrm{N}_{\mathrm{i}} / \mathrm{N}_{\mathrm{p}}=0.2$ ), significant structural changes in the HAZ of both metals are observed. The degeneration of the ferrite's Widmanstatten figures begins because of the formation of smaller and equiaxial subgrains, an increase in the dispersity of the distribution of ferrite and perlite occurs in the main metal $17 \mathrm{G} 1 \mathrm{SU}$ and $09 \mathrm{G} 2 \mathrm{FB}$. 


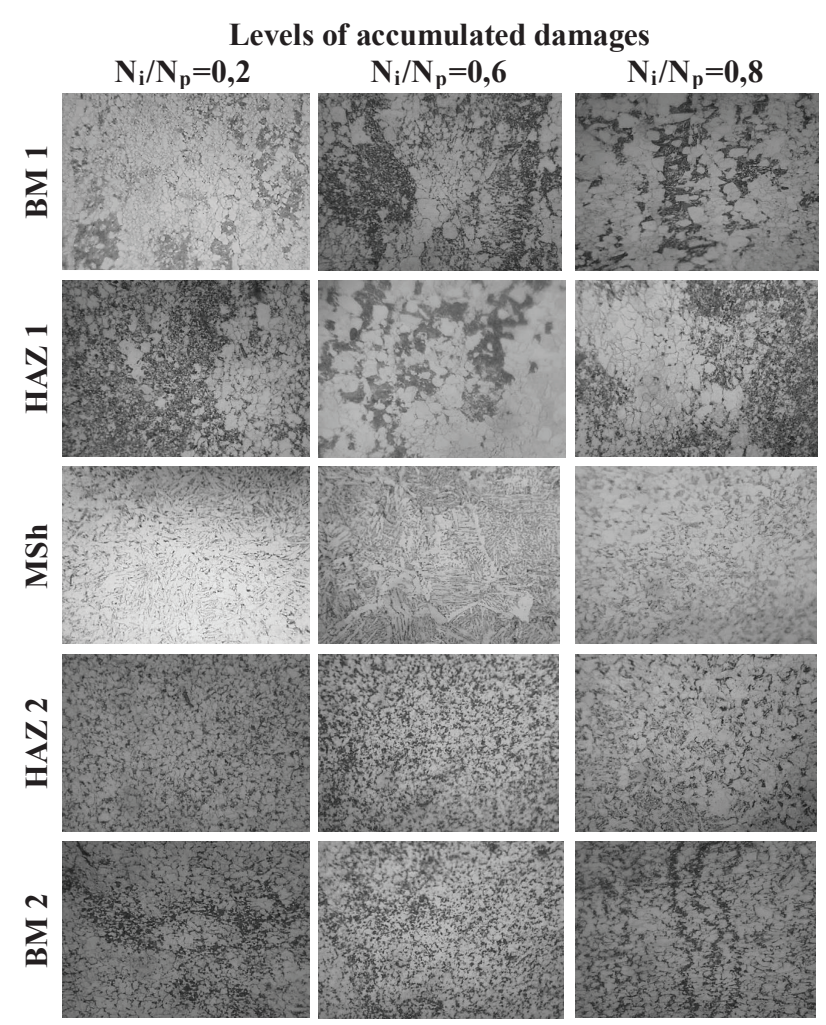

Fig. 3. Change in the microstructure of steel along the welded joint cross-section with accumulation of cyclic damage, $200^{\mathrm{x}}$ :

BM 1 - the basic metal of steel 09G2FB;

HAZ 1 - zone of thermal impact from steel 09G2FB;

MSh - metal of the central zone of a joint;

HAZ 2 - zone of thermal impact from the side of steel $17 \mathrm{G} 1 \mathrm{SU}$;

BM 2 - the basic metal of the steel 17G1SU

With cyclic damages of $0.6 \mathrm{~N}_{\mathrm{i}} / \mathrm{N}_{\mathrm{p}}$, degradation processes affect the dendritic structure of the joint, crushing and leading to equiaxity of its grain. In the remaining zones of the connection grain enlargement is observed. In the main metal, 09G2FB, the discharge bands, which are a sign of irreversible structural changes, are poorly visible.

At a level of $0.8 \mathrm{~N}_{\mathrm{i}} / \mathrm{N}_{\mathrm{p}}$ in the main metal of $17 \mathrm{G} 1 \mathrm{SU}$, the discharge bands are much brighter, signaling the formation of a pre-destruction zone.

In metallographic studies, it was found that because of cyclic loading in the structure of the welded joint, its complete rearrangement took place. First of all, this refers to the morphology of the carbide phase. Due to the production process high-strength strips steel in the initial state had a structure in which the lines of cement particles of the globular shape were clearly identified. They were called in [4] jets of a peculiar plastic flow, and "convenient structural labels" decorating these lines. The directivity of such lines in the structure of the base metals of the compound in the initial state indicates a correspondence between the natures of the plastic deformation of the material to the laminar (-layered) flow regime. In this case, the laminated nature of the plastic flow of material with increasing degree of cyclic damage could be identified only in the surface volumes of the metal.
In the process of cycling in the near-surface layers of the basic metal regions, structural features were also observed. They looked like vortex patterns in the form of closed and open rings and ellipses, which were sufficiently detectable in this structure by the method of optical metallography. The maximum geometric dimension of such a pattern in the cross section did not exceed $25 \mu \mathrm{m}$, and the surface layers themselves had a thickness of 20 to $60 \mu \mathrm{m}$.

Earlier in [4-9], the cause of the formation of vortex patterns in the structure was established. It was caused by the appearance at a certain time of loading of unintended torque in the surface layers of the material being deformed. This is a direct proof of the presence in these layers of explicit signs of a rotational vortex flow, which is associated with special test conditions in which the surface layers are located.

According to [9], plastic deformation arises immediately in several regions of boundaries. Both the cessation of the development of localized flow certain regions and the beginning of the other regions propagation (perpendicular to the loading axis) in the form of bands of different sizes (shape of ellipse) are possible in the early stages of loading.

At the levels of accumulated damages in the range from $0.2 \mathrm{~N}_{\mathrm{i}} / \mathrm{N}_{\mathrm{p}}$ to $0.6 \mathrm{~N}_{\mathrm{i}} / \mathrm{N}_{\mathrm{p}}$, vortex motions in the base metals of the dissimilar weld joint are found. They are created by the propagation of plastic fronts, which move in the region of the action of maximum stresses. However, the direction of motion changes periodically because of stress relaxation in the regions of plastic deformation. After the process of plastic fronts joining coming from opposite surfaces, the development of the second and subsequent layers of plastic deformation, localized in zigzag strips begins. The analogy was found for the localization of plastic deformation inside the strip with the formation of a system of conjugate bands of a smaller scale. It was found that not only individual fragments confined by localization bands of a smaller scale but also their conglomerates are involved in the vortex motion. In the process of cyclic damages accumulation in the microstructure of welded joints different zones, a significant distortion of the crystal takes place. In addition, defects of a crystalline structure arise. At the initial stages of damages accumulation, stress relaxation in ferritic grains is carried out by dislocation sliding and subsequent grain-boundary slip. With an increase in the number of cycles, the relaxation of internal stresses is realized by splitting the individual boundaries of the ferrite grains, or by the initiation of microcracks, mainly in a less strong metal.

Fig. 4 shows that with an increase in the number of loading cycles in a less strong metal of a dissimilar welded joint (base metal - steel 17G1SU), microstress accumulation takes place. It determines the formation in the microstructure of this zone and in the region of the «fault strips». The process of deformation in this case can be attributed to nanocrystalline, because it occurs by the displacement of individual grains of the layers.

On the contrary, in the basic metal of stronger steel (steel 09G2FB), transformation (or recoil) of microstresses is observed. This metal behaves similarly 
to the metal of thermal influence zone located next to it. Separate areas of such a heterogeneous weld joint are under the influence of internal stress relaxation mechanisms. It was noted that at the pre-destruction stage $\left(0.8 \mathrm{~N}_{\mathrm{i}} / \mathrm{N}_{\mathrm{p}}\right)$ the microstress growth manifests itself in all characteristic regions of the welded joint.

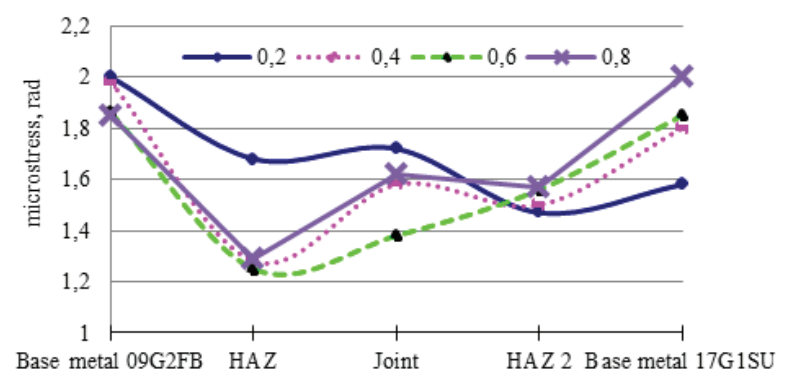

Fig. 4. Changes in the values of microstress over the section of the welded joint from the level of accumulated cyclic damages

With an increase in the number of loading cycles, the value of microdeformation hardly increases in the metal of the thermal influence zone of the less strong metal (steel 17G1SU), which is part of the welded joint, due to accumulation of microstresses. At the same time, the base metal from the same steel is in a more stressed state than the other bond zones, which causes characteristic damages in this zone. In the joint, the microdeformations tend to decrease only to the level of accumulated cyclic damages of $0.6 \mathrm{~N}_{\mathrm{i}} / \mathrm{N}_{\mathrm{p}}$. At the stage of prefracture of the welded joint in this opposite area, microdeformations growth is noted.

The averaged values of the dislocation density in the characteristic regions of the dissimilar welded joint were determined by calculation methods. The dynamics of the change in the process of low-cycle fatigue is shown in Fig. 5. It is obvious that an increase in the level of accumulated damages leads to significant changes in the dislocation structure of materials in the characteristic zones of welded joints.

On graphical dependencies, in almost all regions of the welded joint, a decrease in the density of dislocations is observed, except of the zone of the base less strong metal, where the growth of microstresses was previously observed. The maximum density of dislocations is established at the pre-destruction stage in steel grade $17 \mathrm{G} 1 \mathrm{SU}$, which was caused by a decrease in the distance between dislocations and an increase in the average value of interdislocation interaction forces.

At a level of accumulated damage of $0.8 \mathrm{~N}_{\mathrm{i}} / \mathrm{N}_{\mathrm{p}}$ in the structure of the HAZ metal of less stable steel, an "ensemble of strongly interacting dislocations" [10], with its inherent collective effects, is localized. Their role in the process of metal accumulation of damage in the system "defects - dislocations" becomes the main one.

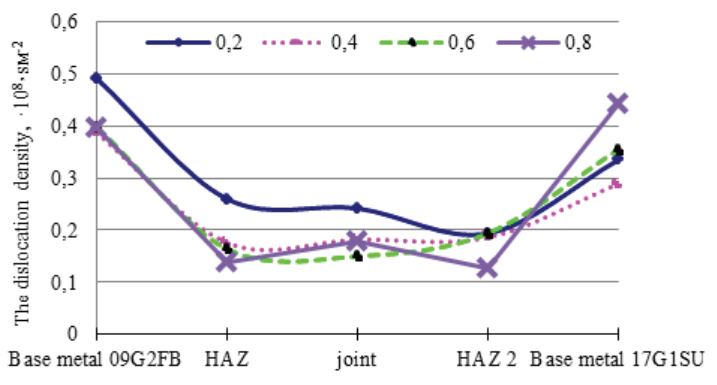

Fig. 5. Distribution of dislocation density along the welded joint cross-section at different levels of cyclic damage

Earlier, in $[11,12]$, the dependence of the energy of dislocation substructures on the degree of ordering of dislocation location was established. The ordering of the dislocation location in the characteristic zones of the welded joint with the accumulation of fatigue damage causes reduction of the long-range field of substructures by elastic dislocation fields Because of the chemical and structural-mechanical inhomogeneity throughout the volume of the welds. In this case, the total energy of the dislocation system decreases.

It is found that the formation of ordered dislocation formations inevitably leads to a decrease in the energy of the "new" structures in comparison with the energy of the "old" structures even with equal degrees of deformation or dislocation densities.

Along with changes in the structural and mechanical state of welded joints under fatigue damage conditions, a change in the mechanism of their destruction from viscous transcrystalline to brittle fracture occurs. Embrittlement presents an increased danger, since the destruction of a welded structure in this case can occur at stresses less than the yield strength, in the form of spontaneous propagation of a crack under the action of the stored elastic energy accumulated by the structure. The changes in the nature of welded joints fracture during the cyclic loading is shown in Fig. 6 in the form of microfractograms of the samples fractures surfaces of the most dangerous section of the welded joint. These are the boundaries of the metal between the zone of thermal influence of the less strong weld metal (steel grade $17 \mathrm{G} 1 \mathrm{SU}$ ) and the base metal (at multiplicity of $200^{\mathrm{x}}$ and $2000^{\mathrm{x}}$ ). Analysis of fracture surfaces was carried out in two regions: the central part of the fracture (1/2 of the sample thickness) and the surface surface of the sample disruption surface (not more than $1 / 8$ of the sample thickness from the surface).

The nature of the fracture of the dangerous zone of the welded joint obtained at room temperature not subjected to cyclic loads was identified as viscous. Cyclic loading of the sample to the level of accumulated damage $\mathrm{N}_{\mathrm{i}} / \mathrm{N}_{\mathrm{p}}=0.2$ leads to the appearance of bundles clearly visible in the investigated region with an increase of 200x. They have the form of transverse grooves (dips), the formation of which is associated with the presence of elongated nonmetallic inclusions in the type of sulphides [13]. A small fraction of the surface of the fracture is occupied by large pits, in which particles of the sulfide type and of oxides of various sizes also found. The central part of the fracture surface is also 
covered with pits, but their dimensions are much smaller than in the near-surface zone. In the pits, sometimes the presence of particles of a nonmetallic nature can be observed.

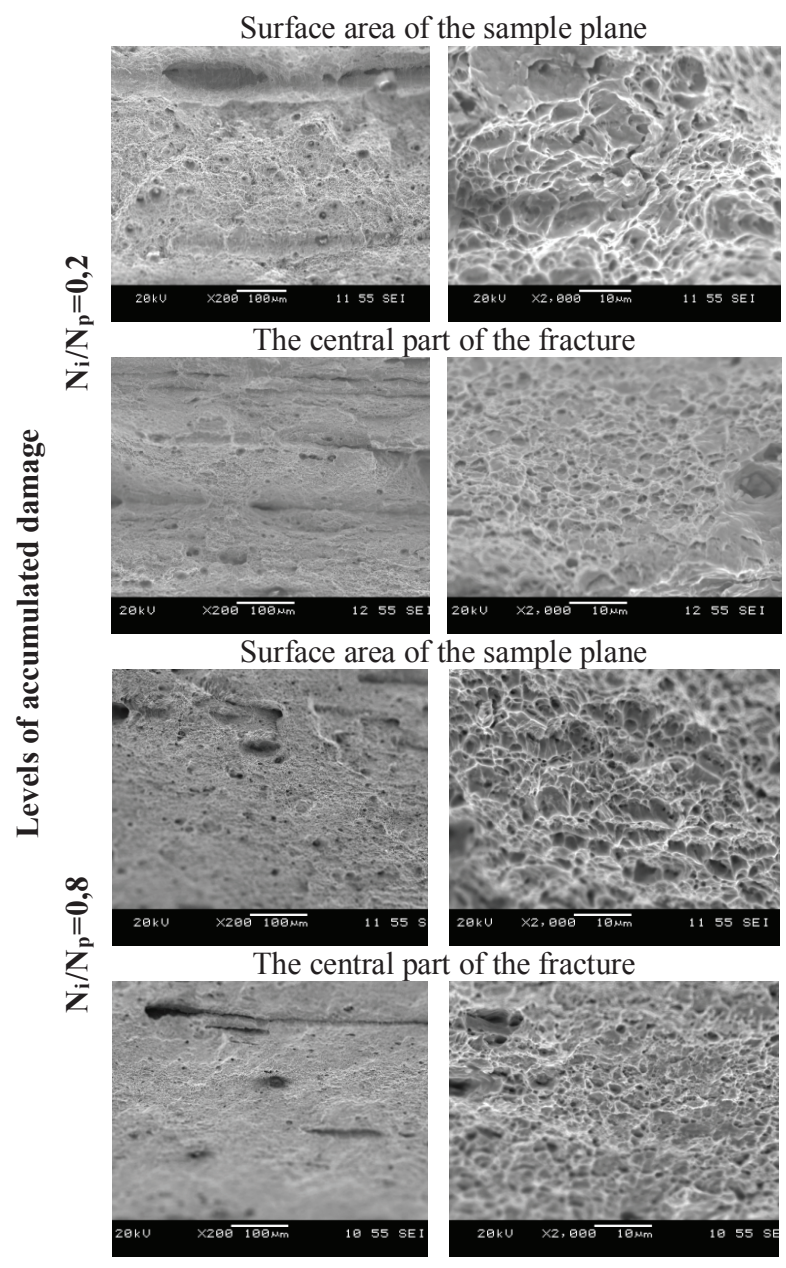

Fig. 6. Microfractogram of the surfaces of specimen fractures in the region of a heterogeneous weld joint at the boundary of the heat-affected zone and the base metal of the less stable $17 \mathrm{G} 1 \mathrm{SU}$ steel at different levels of accumulated damage

With an increase in the number of loading cycles from $0.2 \mathrm{~N}_{\mathrm{i}} / \mathrm{N}_{\mathrm{p}}$ to $0.8 \mathrm{~N}_{\mathrm{i}} / \mathrm{N}_{\mathrm{p}}$, the microstructure of the fracture surface becomes more smooth, the longitudinal grooves (dips) are less deep. The surface of destruction now has a quasi-brittle character, which is clearly expressed with a large increase. The fracture of the central zone contains the areas of separation and fatigue grooves. All illustrations confirm that cyclical fracture is inherent in the combination of fatigue grooves and intergranular fracture.

\section{Conclusions}

Complex metallographic analysis including X-ray diffraction analysis revealed structural changes in the metal of dissimilar welded joints under cyclic damage, distribution of microhardness, degree of distortion of the crystal lattice as the duration of cyclic loading increased. In the process of cyclic loading, structures reminiscent of vortex patterns in the form of closed and open rings and ellipses at the level of optical metallography capabilities are revealed in the near-surface layers of the main metal regions. With an increase in the number of loading cycles at the microscale level, microstresses accumulate in a less strong metal, which leads to the formation of discharge bands in the region adjoined to it.

The dislocation density distribution across the crosssection of a dissimilar welded joint is obtained, which, qualitatively, does not change with increasing number of cycles before failure, which is an important result for understanding the scale hierarchy of the emerging energy dissipation processes. In the field of low-cycle fatigue, meso- and macro-scale levels are involved, when rotations play the main role, and processes do not associated with dislocations. An in-depth analysis of the revealed regularities is made and recommendations are given. In order to describe the plastic deformation in the case of cyclic damage to welded joints, it is necessary to take into account the misorientation of structures based on the disclination theory. In fact, it is justified that exactly the rotations of the crystal cell determine the processes of intensive accumulation of damages in the region of low-cycle fatigue.

\section{References}

1. V.A.Skudnov, M.K. Chegurov, Tehnolog. Met. 3, 6-8 (2008)

2. I.R. Kuzeev, E.V Poyarkova, Neftegaz. Delo, 9, 8086 (2011)

3. E.V. Poyarkova, I.R. Kuzeev, Vest. Tambov. Uni., 18, 1676-1678 (2013)

4. V.A. Bataev, NGTU, 404 (2002)

5. A.A. Bival'kevich, A.A. Bataev, V.G. Burov, V.A. Bataev, S.V. Hlebnikov, Polzunov. vest., 2, 44-46 (2005)

6. V.E. Panin, V.V. Kibitkin, V.S. Pleshanov, Ju.I. Pochivalov, N.A. Lebedeva, Fiz. Mezomeh., 7, 385-388 (2004)

7. A.N. Savkin, V.P. Bagmutov, V.I. Vodop'janov, Izvest Vuz. Mash., 12, 6-14 (2007)

8. A.A. Shanjavskij, A.L. Nikitin, T. Palin-Luc, C. Bathias, Fiz. Mezomeh., 17, 59-68 (2014)

9. V.A. Romanova, R.R. Balohonov, Tjazh. Mash., 9, 27-32 (2010)

10. V.V. Rybin, Metallurgija, (Moscow, 1986)

11. Je.V. Kozlov, V.A. Starenchenko, N.A. Koneva, Metally., 6, 152-161 (1993)

12. N.A.Koneva, Sorosov. Obrazov. Zhur., 6, 99-107 (1996)

13. E.V. Poyarkova, I.R. Kuzeev, Neftegaz. Delo, 6, 239-244 (2008) 